\title{
Supporting Information for Nanoscale particle motion reveals polymer mobility gradient in nanocomposites
}

\author{
Erkan Senses $^{1}$, Suresh Narayanan ${ }^{2}$, Antonio Faraone ${ }^{3}$ \\ ${ }^{1}$ Department of Chemical and Biological Engineering, Koc University, Istanbul 34450, Turkey \\ ${ }^{2}$ Advanced Photon Source, Argonne National Laboratory, Argonne, Illinois 60439, USA \\ ${ }^{3}$ NIST Center for Neutron Research, Gaithersburg, Maryland 20899, USA
}

\section{Sample preparation}

The polymer nanocomposite (PNC) samples with bare NPs was prepared by dissolving PEO in acetonitrile at $30 \mathrm{mg} / \mathrm{ml}$ and adding NPs into polymer solution followed by sonication for $20 \mathrm{~min}$. The solution was then dried under a fume hood for $\approx 12 \mathrm{~h}$ and vacuum-annealed at $363 \mathrm{~K}$ for 2 days and then at $393 \mathrm{~K}$ for 1 day ensure the removal of residual solvent. The PMMA coated NPs were prepared by adsorption of PMMA from its solution in acetonitrile followed by centrifugation/washing steps described in a previous work ${ }^{1}$. The PNCs with PMMA coated NPs were prepared using a protocol similar to bare NP PNC preparation.

\section{Small-angle neutron scattering}

Small-angle neutron scattering (SANS) was performed on beam line NGB30 at the NIST Center for Neutron Research (NCNR, Gaithersburg, MD). Samples sandwiched between quartz windows were molten under vacuum at $363 \mathrm{~K}$ for $15 \mathrm{~min}$ and then tightened to obtain final thicknesses of $\approx 0.5$ $\mathrm{mm}$. The samples were hold at $363 \mathrm{~K}$ to ensure they are molten and then the measurements were performed after 20 min thermal equilibration at the desired temperatures, all above the melting temperature of $\mathrm{PEO}, T_{m, P E O} \approx 338 \mathrm{~K}$. Four configurations with sample-to-detector distances of $(1,4$ and $13 \mathrm{~m}$, without and with lenses) were used to cover $Q$-range from $\approx 0.001 \AA^{-1}$ to $0.5 \AA^{-1}$. All scattering profiles were corrected for background, empty cell and sample transmission to get 1-D isotropic scattering patterns. 

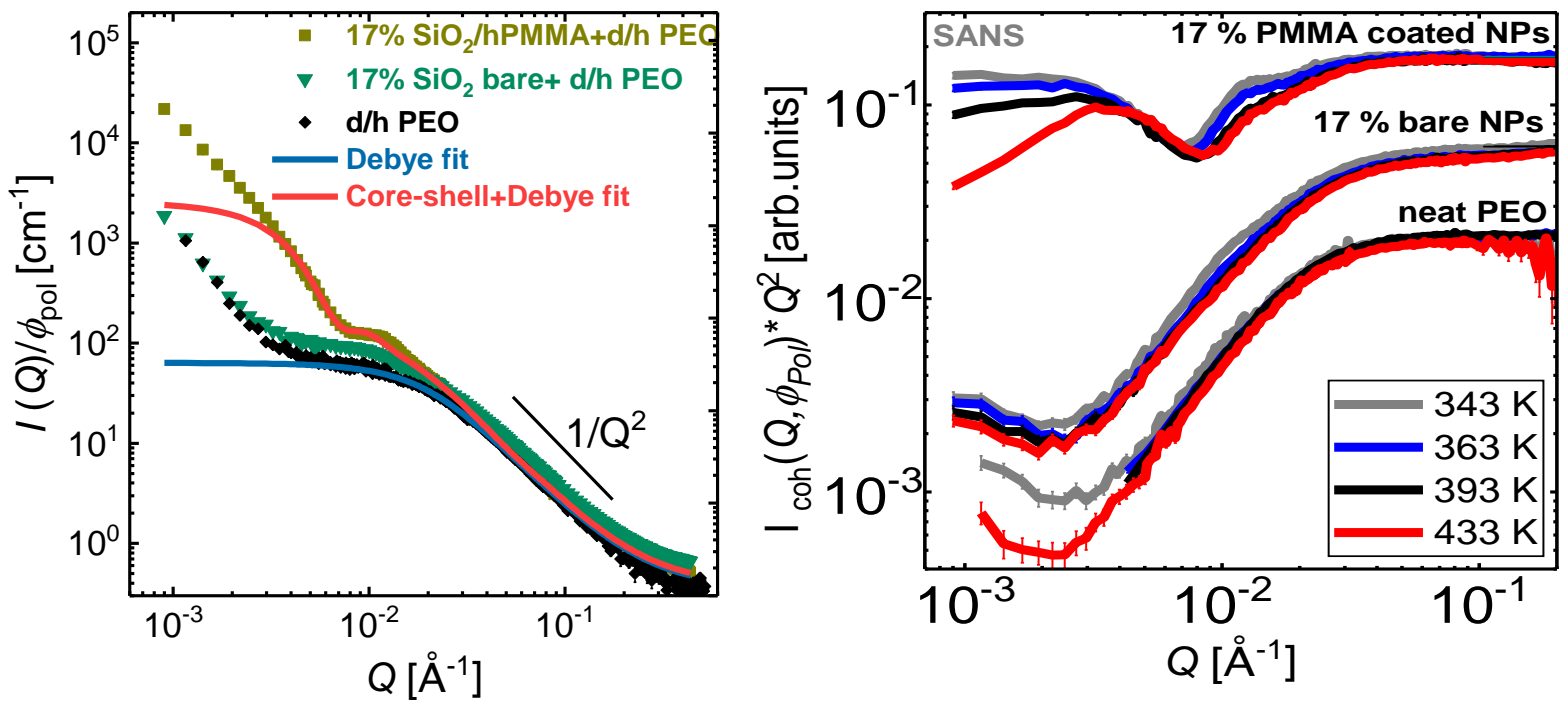

Figure S1. (Left) SANS profiles of the particle-free PEO $(h / d: 49 / 51)$ matrix, the nanocomposites with $17 \%$ bare silica in PEO ( $h / d: 49 / 51)$ and the nanocomposite with $17 \%$ h-PMMA coated silica nanoparticles in PEO ( $h / d$ : 49/51) at $343 \mathrm{~K}$. The data are normalized by the polymer volume fraction. The solid and dashed lines are the Debye function fit to the neat PEO and the sum of core-shell and Debye functions fit to the nanocomposite with $17 \%$ PMMA coated silica. (Right) Kratky plots for the neat PEO, PNC with bare NPs and PNC with PMMA coated $\mathrm{SiO}_{2}$ NPs. Sample sets are shifted vertically for better visual comparison.

Figure S1 shows the SANS results for the neat polymer and the composites at $343 \mathrm{~K}$. The data are normalized by the polymer volume fraction $\left(\phi_{p o l}\right)$. Three different regimes are immediately noticed: (i) At $Q>\approx 0.04 \AA^{-1}$, all data converge to give scaling $I(Q) \sim Q^{-2}$ at the high $Q$ due to the single chain form factor of PEO in the mixture of $d$-PEO and $h$-PEO. The Kratky plots, $Q^{2} I(Q) v s . Q$, obtained after subtracting the incoherent background (the figure on the left) show well-defined plateau in this regime, suggesting that the Gaussian statistics of PEO is retained in the composites.

(ii) At $\approx 0.004 \AA^{-1}>Q>\approx 0.04 \AA^{-1}$, the profile of the composite with PMMA bound layer shows a well-defined feature around $\approx(0.007-0.015) \AA^{-1}$ that is absent in both the neat polymer and the bare 
particle composites. This manifests in the Kratky plots as rather sharp peaks with intensity decreasing with temperature. The location of the peak, $Q^{*} \approx 2 \pi / D \approx 0.01 \AA^{-1}$, matches with the lengthscale of the particle core. Such Kratky peak in contrast-matched homopolymer-nanoparticle mixtures have been observed previously in other attractive nanocomposite systems ${ }^{2}$ and attributed to excess scattering due to bound polymer layer that can originate from various sources such as distortion of chain packing near neutral interfaces, SLD mismatch between core and matrix, and compositional mismatch of $d$ and $h$ chains near solid interface ${ }^{3-5}$; these effects are important mostly in the small particle limit $R \approx R_{g}$, which is different than the case presented in our work. Furthermore, this feature is suppressed significantly in the bare NP-PEO system; therefore, the Kratky peak is due to an enhanced scattering contrast intentionally created by the $h$-PMMA bound layer.

The profiles for the composites with $h$-PMMA bound particles are well described by a combination of Debye and core shell form factors (Fig 1) for data $Q>0.004 \AA^{-1}$ when the SLDs of the core and the matrix were matched and the known parameters, such as $R_{g}$ of the matrix ( $7.67 \mathrm{~nm} \pm 0.05 \mathrm{~nm}$ ) obtained from the Debye form factor fit to neat PEO (solid curve in Fig 1), particle size ( $R=24.3 \mathrm{~nm}$ ) and their polydispersity $(0.31)$. The resulting shell thicknesses of for the PMMA layer is $\approx 10 \mathrm{~nm}$ approximately $2 R_{\mathrm{g}}$ of the polymer. The resulting $\mathrm{SLD}=1.04 \times 10^{-6} \AA^{-2}$ of PMMA was found to be in good agreement with the bulk $h$-PMMA chains, suggesting that mixing of PEO and bound PMMA at $343 \mathrm{~K}$ is negligible. The decrease of the peak intensity of the PMMA shell (in Kratky plot) with temperature is likely due to enhanced intermixing of PMMA and PEO at elevated temperatures, which cause a reduction in scattering contrast.

(iii) At $Q<\approx 0.004 \AA^{-1}$, the excess scattering beyond the core-shell contribution of the bound layer is possibly due to a larger scale composition fluctuations of $h$ PEO and $d$ PEO chains since the same 
intensity upturn is also seen for particle-free neat matrix (therefore the fits were performed for Q lower than $\left.0.004 \AA^{-1}\right)$.

\section{X-ray photon correlation spectroscopy}
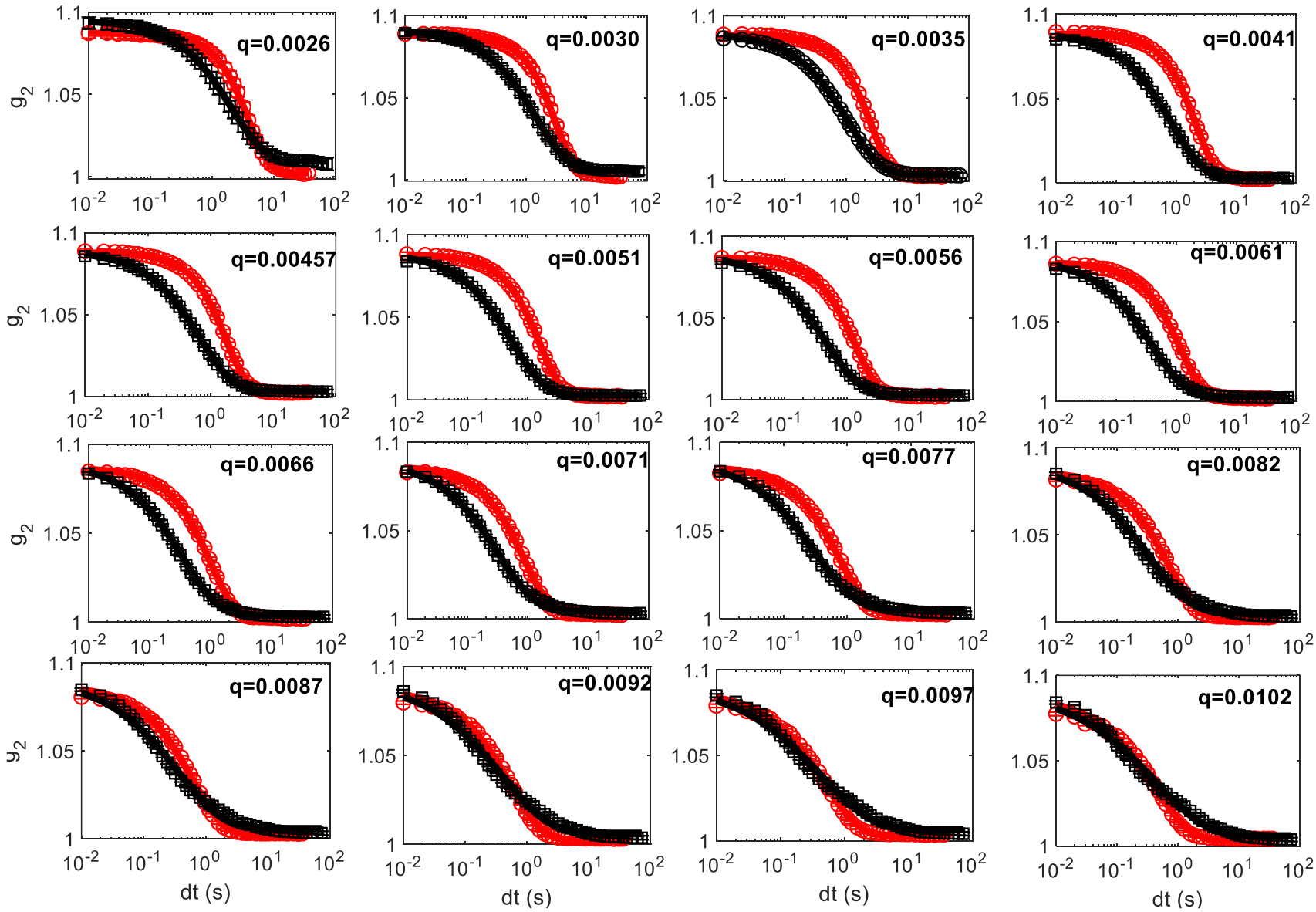

Figure S2. Comparison of the correlation functions for bare NPs (red circles) and PMMA coated NPs (black squares) at different wave-vectors at $393 \mathrm{~K}$ and the corresponding fits to stretched exponential decay with global contrast factors. 


\section{Average Relaxation time}

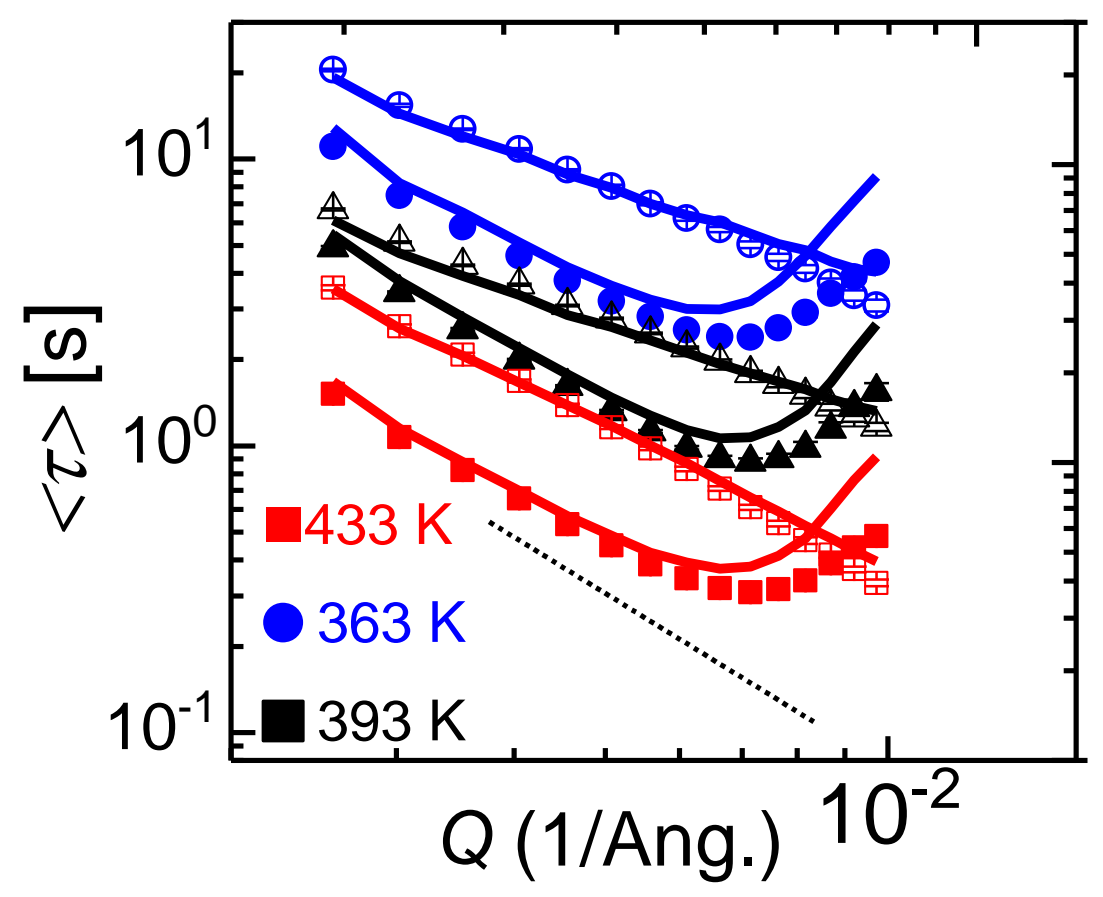

Figure S3. Comparison between the relaxation time $\tau$ (symbols) and the average relaxation time, $\langle\tau\rangle=(\tau / \beta) \Gamma(1 / \beta), \Gamma$ being the gamma function (lines). The open and filled symbols are for the bare NPs and the PMMA coated NPs, respectively.

\section{Structural stability during measurements}

Well-dispersion of the PMMA coated silica nanoparticles in PEO at elevated temperatures and their structural stability were shown (via electron microscopy and SAXS/SANS) elsewhere ${ }^{1,6}$. Here, the stability of the NP dispersion during the XPCS measurements was verified by the static SAXS data collected for each measurement. Figure S4a compares ten SAXS profiles of the PNC with PMMA coated NPs in PEO obtained during a measurement at $433 \mathrm{~K}$ (The curves are shifted vertically for clarity). The profiles are identical, the NP structure did not change during the measurements; therefore, the observed dynamical behavior reported is not related to changes in structure. In addition, Figure S4b compares the SAXS curves obtained during two subsequent XPCS runs, which were performed on different locations of the sample. The identical profiles further confirms the homogeneity of the NP dispersion in the matrices. 

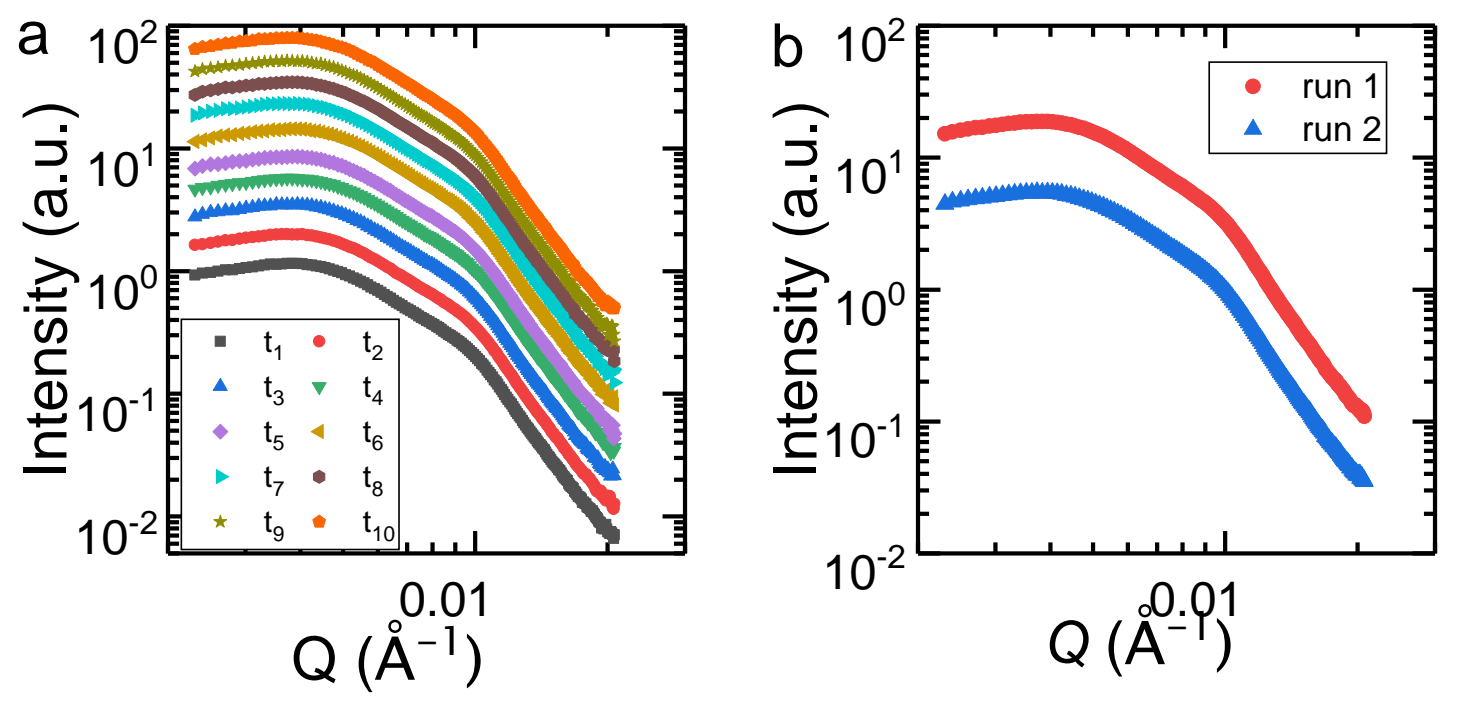

Figure S4. (a) Comparison of ten SAXS profiles obtained during the XPCS measurements on PMMA coated NPs in PEO at $433 \mathrm{~K}$. (b) Comparison of the SAXS profiles obtained for two subsequent XPCS runs performed on different locations of the sample.

\section{Note}

Error bars represent one standard deviation throughout this work.

\section{References}

1. Senses, E.; Faraone, A.; Akcora, P., Microscopic Chain Motion in Polymer Nanocomposites with Dynamically Asymmetric Interphases. Sci. Rep. 2016, 6, 29326.

2. Jouault, N.; Crawford, M. K.; Chi, C.; Smalley, R. J.; Wood, B.; Jestin, J.; Melnichenko, Y. B.; He, L.; Guise, W. E.; Kumar, S. K., Polymer Chain Behavior in Polymer Nanocomposites with Attractive Interactions. ACS Macro Lett. 2016, 5, 523-527.

3. Banc, A.; Genix, A.-C.; Dupas, C.; Sztucki, M.; Schweins, R.; Appavou, M.-S.; Oberdisse, J., Origin of Small-Angle Scattering from Contrast-Matched Nanoparticles: A Study of Chain and Filler Structure in Polymer Nanocomposites. Macromolecules 2015, 48, 6596-6605.

4. Nusser, K.; Neueder, S.; Schneider, G. J.; Meyer, M.; Pyckhout-Hintzen, W.; Willner, L.; Radulescu, A.; Richter, D., Conformations of Silica-Poly(Ethylene-Propylene) Nanocomposites. Macromolecules 2010, 43, 9837-9847.

5. Genix, A.-C.; Tatou, M.; Imaz, A.; Forcada, J.; Schweins, R.; Grillo, I.; Oberdisse, J., Modeling of Intermediate Structures and Chain Conformation in Silica-Latex Nanocomposites Observed by Sans During Annealing. Macromolecules 2012, 45, 1663-1675.

6. Senses, E.; Isherwood, A.; Akcora, P., Reversible Thermal Stiffening in Polymer Nanocomposites. ACS Appl. Mater. Interfaces 2015, 7, 14682-14689. 